\title{
Cluster electric current density measurements within a magnetic flux rope in the plasma sheet
}

\author{
J. A. Slavin, ${ }^{1}$ R. P. Lepping, ${ }^{1}$ J. Gjerloev, ${ }^{1}$ M. L. Goldstein, ${ }^{1}$ D. H. Fairfield, ${ }^{1}$ \\ M. H. Acuna, ${ }^{1}$ A. Balogh, ${ }^{2}$ M. Dunlop, ${ }^{2}$ M. G. Kivelson, ${ }^{3}$ K. Khurana, ${ }^{3}$ A. Fazakerley, ${ }^{4}$ \\ C. J. Owen, ${ }^{4}$ H. Reme, ${ }^{5}$ and J. M. Bosqued ${ }^{5}$ \\ Received 8 October 2002; revised 19 January 2003; accepted 4 March 2003; published 2 April 2003.
}

[1] On August 22, 2001 all 4 Cluster spacecraft nearly simultaneously penetrated a magnetic flux rope in the tail. The flux rope encounter took place in the central plasma sheet, $\beta_{\mathrm{i}} \sim 1-2$, near the leading edge of a bursty bulk flow. The "time-of-flight" of the flux rope across the 4 spacecraft yielded $\mathrm{V}_{\mathrm{x}} \sim 700 \mathrm{~km} / \mathrm{s}$ and a diameter of $\sim 1 \mathrm{R}_{\mathrm{e}}$. The speed at which the flux rope moved over the spacecraft is in close agreement with the Cluster plasma measurements. The magnetic field profiles measured at each spacecraft were first modeled separately using the Lepping-Burlaga forcefree flux rope model. The results indicated that the center of the flux rope passed northward (above) s/c 3, but southward (below) of s/c 1,2 and 4. The peak electric currents along the central axis of the flux rope predicted by these single-s/c models were $\sim 15-19 \mathrm{nA} / \mathrm{m}^{2}$. The 4-spacecraft Cluster magnetic field measurements provide a second means to determine the electric current density without any assumption regarding flux rope structure. The current profile determined using the curlometer technique was qualitatively similar to those determined by modeling the individual spacecraft magnetic field observations and yielded a peak current density of $17 \mathrm{nA} / \mathrm{m}^{2}$ near the central axis of the rope. However, the curlometer results also showed that the flux rope was not force-free with the component of the current density perpendicular to the magnetic field exceeding the parallel component over the forward half of the rope, perhaps due to the pressure gradients generated by the collision of the BBF with the inner magnetosphere. Hence, while the single-spacecraft models are very successful in fitting flux rope magnetic field and current variations, they do not provide a stringent test of the force-free condition. INDEX TERMS: 2744 Magnetospheric Physics: Magnetotail; 2788 Magnetospheric Physics: Storms and substorms; 2740 Magnetospheric Physics: Magnetospheric configuration and dynamics. Citation: Slavin, J. A., et al., Cluster electric current density measurements within a magnetic flux rope in the plasma sheet, Geophys. Res. Lett., 30(7), 1362, doi:10.1029/2002GL016411, 2003.

\footnotetext{
${ }^{1}$ Laboratory for Extraterrestrial Physics, NASA Goddard Space Flight Center, Greenbelt, Maryland, USA..

${ }^{2}$ Space and Atmospheric Physics, Imperial College, London, UK..

${ }^{3}$ Institute of Geophysics and Planetary Physics, University of California, Los Angeles, California, USA..

${ }^{4}$ Mullard Space Science Laboratory, Holmbury St. Mary, Dorking, England, UK.

${ }^{5}$ Centre d'Etude Spatiale des Rayonnements, Toulouse, France..
}

Copyright 2003 by the American Geophysical Union. 0094-8276/03/2002GL016411

\section{Introduction}

[2] Plasmoids or plasmoid-type magnetic flux ropes moving anti-sunward in the distant tail have been investigated extensively by the ISEE 3 [Moldwin and Hughes, 1992] and Geotail [Ieda et al., 1998] missions. Isolated examples of magnetic flux ropes in the near-earth plasma sheet were returned by a number of early missions [Elphic et al., 1986; Sergeev et al., 1992; Kivelson et al., 1993]. However, more recently, the Geotail plasma sheet measurements have been surveyed for the presence of magnetic flux by Slavin et al. [2003]. They found that small, $\sim 1-5 \mathrm{R}_{\mathrm{e}}$ diameter, flux ropes are common in the $\mathrm{X} \sim-20$ to $-30 \mathrm{R}_{\mathrm{e}}$ region, occurring at a rate of 1 every $\sim 5 \mathrm{hrs}$. All were associated with high speed plasma sheet flows and they evidenced a strong tendency to be found near the leading edges of earthward or tailward flow events. Slavin et al. termed these flux ropes either bursty bulk flow (BBF)-type or plasmoid-type, depending upon whether they were embedded in earthward or tailward high speed flows, respectively, with similar numbers of flux ropes falling into each category. By comparison, Moldwin and Hughes [1992] found only $\sim 50$ flux flux ropes moving earthward at $\mathrm{X}<$ $-100 \mathrm{R}_{\mathrm{e}}$ out of a total of nearly 600 . The significance of flux ropes in the plasma sheet is that their formation can most readily be understood in terms of simultaneous reconnection at multiple X-lines (MRX) in the near-tail by analogy with the MRX models proposed to explain the rope-like structure of flux transfer events at the dayside magnetopause [see Lee, 1995]. Figure 1 illustrates how simultaneous reconnection at $\mathrm{N}+1 \mathrm{X}$-lines leads to the generation of $\mathrm{N}$ flux ropes (adapted from Slavin et al. [2003]). Multiple X-line models of this sort have been previously used to explain earthward moving flux rope's by Elphic et al. [1986] and Moldwin and Hughes [1994].

[3] The polarity of the flux ropes' core magnetic field is largely determined by the direction of the IMF $\mathrm{B}_{\mathrm{y}}$ as predicted by Hughes and Sibeck [1987]. The idea that a large segment of the strongly thinned cross-tail current layer might become unstable to reconnection is not new. Such a concept is central to tearing mode models of reconnection going back to the 1960's. However, as first argued by Schindler [1974], one of the X-lines will inevitably outpace the others and begin to reconnect first the outer plasma sheet, then the PSBL and, finally, lobe flux tubes where $\mathrm{V}_{\mathrm{A}} \sim 1000 \mathrm{~km} / \mathrm{s}$ or higher [see Hesse et al., 1996]. At that point, everything earthward of the first X-line to reconnect lobe flux tubes will be carried toward the Earth, and all material tailward will be rapidly swept down the tail. Hence, the formation of flux ropes by MRX reconnection of closed field lines is in a sense only a preliminary 


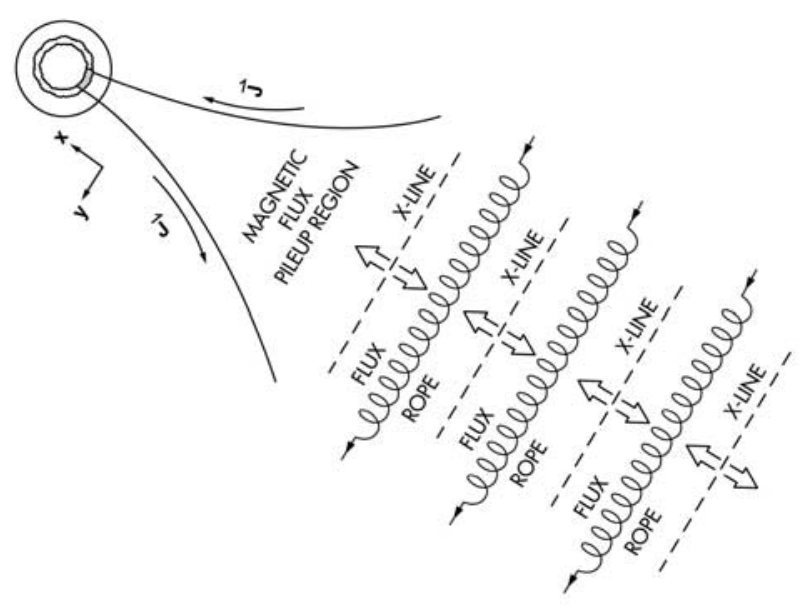

Figure 1. Schematic depiction of the formation of flux ropes as a result of multiple, simultaneous reconnection neutral lines in the X-Y plane (adapted from Slavin et al. [2002]).

event to the open flux reconnection at a single neutral line in the NENL model of substorms [Baker et al., 1996].

\section{August 22, 2001 Flux Rope}

[4] The first Cluster tail "season" was surveyed for the presence of flux rope signatures in a manner similar that applied to the Geotail measurements by Slavin et al. [2003]. Briefly, the Cluster FGM magnetic field measurements [Balogh et al., 1997] taken in or near the plasma sheet were examined in order to identify all \pm or $\mp$ variations in the $\mathrm{B}_{\mathrm{z}}$ component on time scales of seconds to minutes. It was required that GSE $B_{z}$ reach negative values, but not that the perturbation be symmetric about $\mathrm{B}_{\mathrm{z}} \sim 0$. Finally, the events were further screened to eliminate those lacking coincident enhancements of the $\mathrm{B}_{\mathrm{y}}$ and/or $\mathrm{B}_{\mathrm{x}}$ field components in order to separate "ropes" from "loops".

[5] We found only one clear flux rope that was simultaneously sampled by all $4 \mathrm{~s} / \mathrm{c}$. A number of cases were found where 1, 2 or 3 of the Cluster spacecraft were simultaneously in a flux rope, but the probability of having all 4 simultaneous within a flux rope is small, because the diameter of these flux ropes are typically only $1-2 \mathrm{R}_{\mathrm{e}}$ or just 3 to 6 times the Cluster s/c separations. The FGM magnetic field observations between 10:08 and 10:09 on August 22, 2001 are displayed in Figure 2. The Cluster spacecraft were located at GSE $\mathrm{X}=-19, \mathrm{Y}=-3$, and $\mathrm{Z}=-1 \mathrm{R}_{\mathrm{e}}$. and their maximum separations were $\Delta \mathrm{X}=0.26, \Delta \mathrm{Y}=0.29$ and $\Delta \mathrm{Z}=0.30 \mathrm{R}_{\mathrm{e}}$. The Cluster CIS plasma ion and electron measurements [Rème et al., 2001; Johnstone et al., 1997] showed that all $4 \mathrm{~s} / \mathrm{c}$ were deep in the plasma sheet, $\beta_{\mathrm{i}} \sim 1-2$, and the $\mathrm{B}_{\mathrm{x}}$ component of the magnetic field indicated that they were just south of the cross-tail current layer as expected based upon their physical location. All $4 \mathrm{~s} / \mathrm{c}$ show a large amplitude, \pm 15 $n T, B_{z}$ perturbation beginning at 10:08:22-:25 coincident with enhancements in the $\mathrm{B}_{\mathrm{x}}$ and $\mathrm{B}_{\mathrm{y}}$ components. The $\mathrm{B}_{\mathrm{z}}$ perturbation durations are all $\sim 9 \mathrm{~s}$. The $\mathrm{B}_{\mathrm{y}}$ field enhancement at Cluster had the same sense as the upstream IMF at $\mathrm{ACE}$ (not shown). These enhancements in $\mathrm{B}_{\mathrm{x}}$ and $\mathrm{B}_{\mathrm{y}}$ result in an increase in the total field intensity of $\sim 20 \mathrm{nT}$ as shown in the fourth panel of Figure 2. This increase in magnetic pressure briefly reduces the plasma ion beta values to values of $0.2-0.3$ at the center of the flux rope. Finally, it should be noted that this flux rope was located near the leading edge of a BBF which, in turn, was part of a series of such earthward flows associated with a substorm that began a little before 10:00.

[6] Closer inspection of Cluster FGM measurements shows that the $\mathrm{B}_{\mathrm{z}}$ perturbations at $\mathrm{s} / \mathrm{c} 2-4$ precede that observed at $\mathrm{s} / \mathrm{c} 1$ by about $2 \mathrm{~s}$. Given the $0.22 \mathrm{R}_{\mathrm{e}}$ difference in the X coordinates of $\mathrm{s} / \mathrm{c} 1$ and $\mathrm{s} / \mathrm{c} 2-4$ this time delay indicates that the flux rope moved over the tetrahedron with a earthward directed speed $V_{x}=700 \mathrm{~km} / \mathrm{s}$. This speed is consistent with the $600-800 \mathrm{~km} / \mathrm{s}$ speeds observed in the Cluster plasma ion measurements displayed in the bottom panel of Figure 2. Given the $9 \mathrm{~s}$ duration for the flux rope, the implied diameter of this structure is $\sim 1.0 \mathrm{R}_{\mathrm{e}}$. Finally, this flux rope is located in the leading portion of a BBF (N.B., a small substorm was underway in the auroral magnetograms [not shown]) as was very frequently observed to be the case for the large number of such events that have been analyzed using the Geotail measurements [Slavin et al., 2003].

\section{Single S/C Flux Rope Modeling}

[7] In order to model the flux rope signatures observed by the individual spacecraft we will utilize the Lepping-

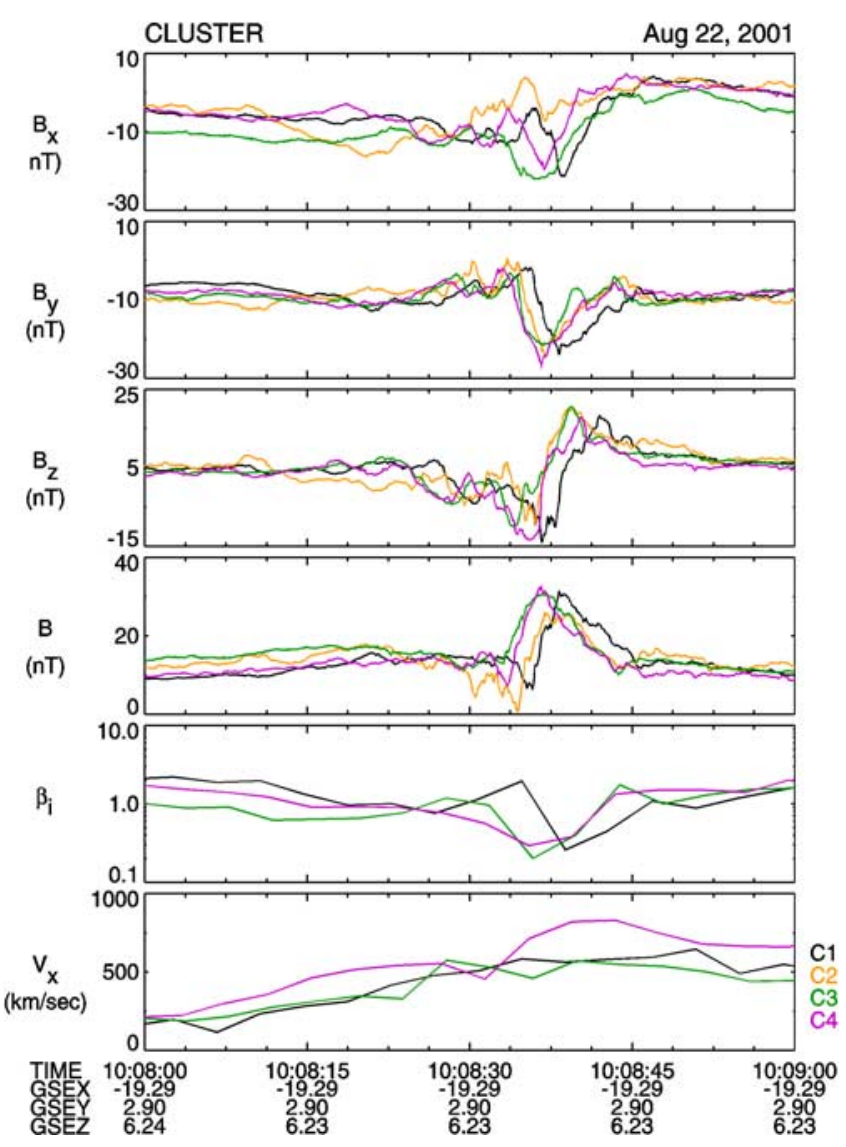

Figure 2. The components of the magnetic field measured at all 4 Cluster s/c are displayed in GSE coordinates along with plasma $\beta_{\mathrm{i}}$ and $\mathrm{V}_{\mathrm{x}}$. Note the $\sim 2$ sec delay between the arrival of the flux rope $B_{z}$ perturbation at s/c 2-4 and s/c 1 . 
Burlaga model [Lepping et al., 1990] that assumes them to be approximately force-free structures and then, in the next section, we will use the data from all $4 \mathrm{~s} / \mathrm{c}$ to test this assumption. Hence, for the single spacecraft modeling, the current density $(\mathbf{J})$ and the magnetic field $(\mathbf{B})$ are parallel or anti-parallel:

$$
\mathbf{J}=\alpha \mathbf{B}
$$

This condition defines the so-called "constant $\alpha$ " class of force-free flux ropes. The structure is assumed to be cylindrically symmetric with the pitch angle of the helical field lines increasing with growing distance from the axis of the rope. The Lepping-Burlaga method fits the Lundquist solution to the magnetic field measurements taken along arbitrary trajectories through the flux rope. A variance analysis is applied to these data in order to establish an approximate rope coordinate system. After transformation into this initial coordinate system, a least-squares fitting is then performed upon the unit normalized, observed magnetic field. The full set of flux rope-fitted parameters are: $B_{O}$, the axial field intensity; $\mathrm{H}$, the handedness of the field twist ( \pm 1 for right/left handedness); $R_{O}$, the radius of the flux rope; $\Phi_{A}, \Theta_{A}$, the longitude and latitude of the rope's axis, respectively; $t_{O}$, the rope center time; and $Y_{O} / R_{O}$ is termed the "impact parameter". Additional details of the procedure can be found in Lepping et al. [1990] and Slavin et al. [2003].

[8] Modeling of the magnetic field profiles obtained by the $4 \mathrm{~s} / \mathrm{c}$ yielded flux rope diameters ranging from 1.08 to $0.96 \mathrm{R}_{\mathrm{e}}$ and predicted peak magnetic fields and current densities at the central axis of the flux rope of 28.9 to 38.9 $\mathrm{nT}$ and 15.1 to $18.9 \mathrm{nA} / \mathrm{m}^{2}$. The $\mathrm{s} / \mathrm{c}$ trajectories inferred from modeling the individual magnetic field profiles had impact parameters of 0.41 to 0.66 with s/c 1,2 , and 4 passing to the north of the central axis of the flux rope and s/ c 3 passing to the south. The elevations of the central axis to the GSE X-Y plane determined ranged from -12 deg to +17 deg. Similarly, the azimuthal angles ran from $202 \mathrm{deg}$ to $270 \mathrm{deg}$ where $90 \mathrm{deg}$ is toward dusk and $270 \mathrm{deg}$ is dawnward. Overall, the quality of the model fits was reasonably good and comparable to those obtained in the Geotail flux rope study described earlier [Slavin et al., 2003].

\section{Curlometer Current Density Measurement}

[9] Access to $4 \mathrm{~s} / \mathrm{c}$ magnetic field measurements enables the computation of the local electric current density vector from the magnetic field gradients [Dunlop et al., 1990]. In order to apply this technique to the August 22, 2001 flux rope, the magnetometers on the Cluster spacecraft have first been inter-calibrated and the current density was then inferred from the nine elements of the magnetic field gradient matrix, $\partial \mathrm{B}_{\mathrm{i}} / \partial \mathrm{x}_{\mathrm{j}}$, to compute the current density vector following the algorithms described by Khurana et al. [1996]. The current density and the magnetic field at the center of the tetrahedron inferred from the field gradients were then used to compute the current density parallel and perpendicular to the local magnetic field as a function of time.

[10] The results of the curlometer analysis of this event are shown in Figure 3. Vertical dashed lines mark the

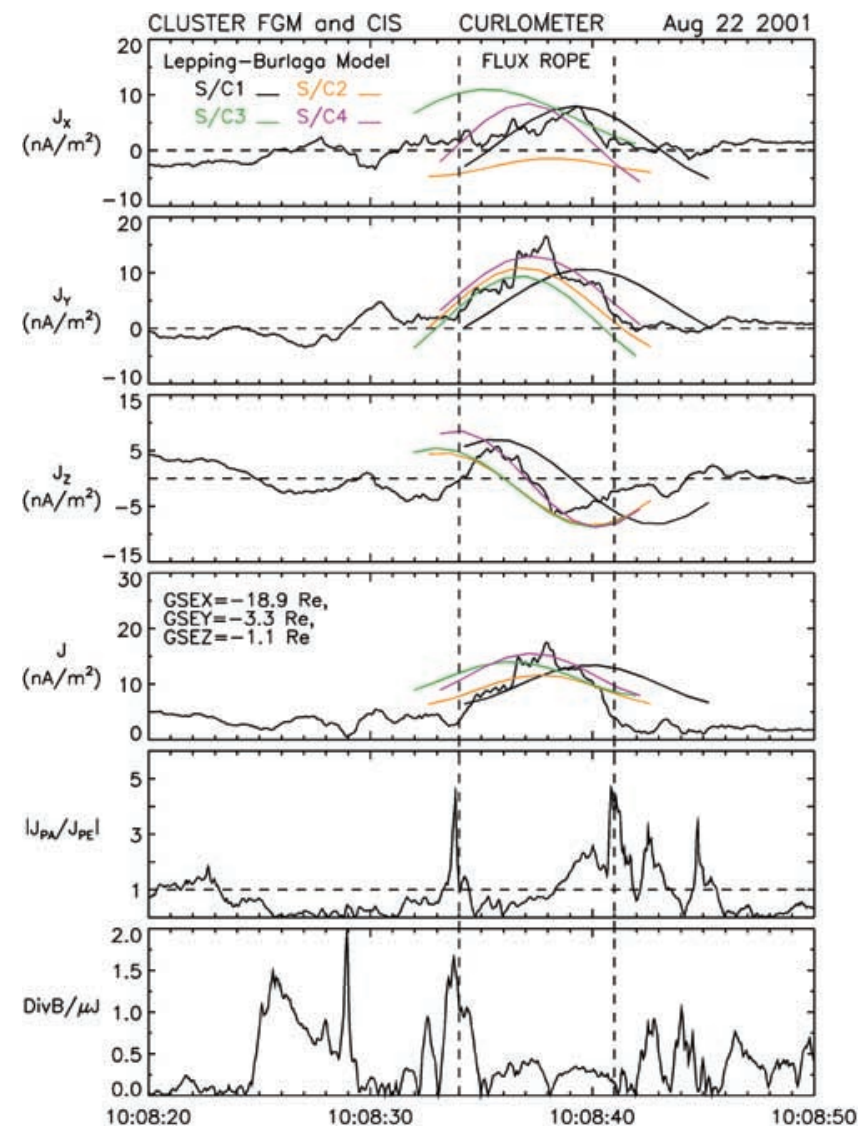

Figure 3. Electric current density determined using the 4 $\mathrm{s} / \mathrm{c}$ curlometer technique and the single s/c Lepping-Burlaga model is displayed. The ratio of the curlometer determined current parallel to the magnetic field to that perpendicular to the magnetic field is presented in the 5 th panel. The error in the curlometer current, taken to be the divergence of $\mathrm{B}$ divided by $\mu_{\mathrm{o}} \mathrm{J}$ is shown in panel 6 .

approximate boundaries of the flux rope in the $4 \mathrm{~s} / \mathrm{c}$ data set. The curlometer determined current is plotted in black alongside the Lepping-Burlaga single s/c model currents in colors corresponding to the individual s/c. As shown the middle of the flux rope is marked by a strong peak in $\mathrm{J}_{\mathrm{y}}$ opposite the direction of the core field of the flux rope in Figure 2. This means that this is a "left-handed" flux rope with the current and the field directions tending to be oppositely directed [e.g., Priest, 1990]. Similarly, the $\mathrm{J}_{\mathrm{z}}$ current exhibits a very clear, relatively symmetric bipolar variation with its sense opposite to that of the $B_{z}$ field variation in Figure 2, again, as expected for a lefthanded flux rope. The peak in the total electric current density measured near the central axis of the flux rope was $17 \mathrm{nA} / \mathrm{m}^{2}$. This is greater than the single $\mathrm{s} / \mathrm{c}$ model current densities along the individual s/c trajectories, but very close to the values predicted by the single s/c models at the center of the flux rope. The fractional error in the current measurement, taken to be of order Div $\mathrm{B} / \mu_{\mathrm{o}} \mathrm{J}$, is displayed in the bottom panel. Despite the diameter of the flux rope being only about 4 times the s/c separations, i.e., $\sim 6400 \mathrm{~km}$ versus $1800 \mathrm{~km}$, the error in the current is reasonably small running between 0 and $25 \%$ over most of the flux rope. 
[11] Finally, we have also computed the current density components parallel and perpendicular to the local magnetic field. Figure 3 includes a plot of the absolute value of the ratio of the parallel to the perpendicular current with a horizontal dashed line marking the level where they are equal. The result indicates that the perpendicular current dominates the parallel current over the first half of the flux rope. It is only in the trailing half of the flux rope that the parallel current dominates as is required for a force-free flux rope, i.e. $\mathrm{J} \times \mathrm{B} \sim 0$. However, judging from the good qualitative agreement between the Lepping-Burlaga model current determinations and the curlometer result in the top panels, it appears that these single-s/c methods can produce good fidelity magnetic field and current density representations even when the structures being modeled deviate significantly from being force-free.

\section{Summary}

[12] Cluster measurements taken on August 22, 2001 included a well-defined magnetic flux rope embedded in the leading edge of an earthward directed plasma sheet flow burst. All $4 \mathrm{~s} / \mathrm{c}$ passed through the structure enabling both the traditional single $\mathrm{s} / \mathrm{c}$ and the new four $\mathrm{s} / \mathrm{c}$ analysis of its properties. Excellent agreement was found between the Cluster plasma velocity measurements and the TOF speed determined from the time delays as the flux rope is swept over the $4 \mathrm{~s} / \mathrm{c}$ confirming that this magnetic structure was frozen into the hot, $\beta_{\mathrm{i}} \sim 1-2$, rapidly flowing, $\mathrm{V}_{\mathrm{x}} \sim 700$ $\mathrm{km} / \mathrm{s}$, plasma sheet. The diameter of the flux rope was $\sim 1 R_{e}$ and it was oriented largely across the plasma sheet with an azimuthal angle to the sunward direction of $227 \mathrm{deg}$ and an elevation angle of 6.8 deg to the GSE X-Y plane. Both the single $\mathrm{s} / \mathrm{c}$ Lepping-Burlaga modeling and the curlometer technique determined that this was a left-handed flux rope and that the peak current density at the center of the rope was $\sim 17 \mathrm{nA} / \mathrm{m}^{2}$. However, the curlometer current density determination showed that the flux rope was not completely force-free as assumed by the single s/c models, but rather that the parallel current was less than the perpendicular current over most of the forward portion of the rope. Hence, while the single-spacecraft models are very successful in fitting flux rope magnetic field and current variations, they do not provide a stringent test of the force-free condition. These deviations from the force-free condition may be due to the macro-scale fluid pressure gradients resulting from the collision between the earthward directed BBF and the inner magnetosphere.

[13] Acknowledgments. The authors wish to thank all of those who contributed to the success of the Cluster Mission. Discussions with D.G. Sibeck are also gratefully acknowledged.

\section{References}

Baker, D. N., et al., Neutral line model of substorms: Past results and present view, J. Geophys. Res., 101, 12,975, 1996.

Balogh, A., et al., Cluster magnetometer team, the cluster magnetic fields investigation, Space Sci. Rev., 79, 65, 1997.

Dunlop, M. W., et al., Configurational sensitivity of multipoint magnetic field measurements, in Proceedings of the International Workshop on Space Plasma Physics: Investigations by Cluster and Regatta, Eur. Space Agency Spec. Publ., ESA SP 306, 23-28, 1990.

Elphic, R. C., et al., Observations of magnetic flux ropes in the magnetotail: FTEs in the plasma sheet?, Geophys. Res. Lett., 14, 648, 1986.

Hesse, M., J. Birn, D. N. Baker, and J. Slavin, MHD simulations of the transition of magnetic reconnection from closed to open field lines, J. Geophys. Res., 101, 10,805, 1996.

Hughes, W. J., and D. G. Sibeck, On the three dimensional structure of plasmoids, Geophys. Res. Lett., 14, 636, 1987.

Ieda, A., S. Machida, T. Mukai, Y. Saito, T. Yamamoto, A. Nishida, T. Terasawa, and S. Kokubun, Statistical analysis of plasmoid evolution with Geotail observations, J. Geophys. Res., 103, 4435, 1998.

Johnstone, A. D., et al., PEACE: A plasma electron and current experiment, Space Sci. Rev., 79, 351, 1997.

Khurana, K. K., et al., Accurate determination of magnetic field gradients from four point vector measurements - part II: Use of natural constraints on vector data obtained from a single spinning spacecraft, IEEE Trans. Magn., 32, 5193, 1996.

Kivelson, M. G., et al., The Galileo Earth encounter: Magnetometer and allied measurements, J. Geophys. Res., 98, 11,299, 1993.

Lee, L. C., A review of magnetic reconnection: MHD models, in Physics of the Magnetopause, Geophys. Monogr. Ser., vol. 90, edited by P. Song, B. U. O. Sonnerup, and M. F. Thomsen, pp. 139-153, AGU, Washington, D. C., 1995.

Lepping, R. P., J. A. Jones, and L. F. Burlaga, Magnetic field structure of interplanetary magnetic clouds at $1 \mathrm{AU}, J$. Geophys. Res., 95, 11,957, 1990.

Moldwin, M. B., and W. J. Hughes, On the formation and evolution of plasmoids: A survey of ISEE 3 geotail, J. Geophys. Res., 97, 19,259, 1992.

Moldwin, M. B., and W. J. Hughes, Observations of earthward and tailward propagating flux rope plasmoids: Expanding the plasmoid model of geomagnetic substorms, J. Geophys. Res., 99, 183, 1994.

Priest, E. R., The equilibrium of magnetic flux ropes, in Physics of Magnetic Flux Ropes, Geophys. Monogr. Ser., vol. 58, edited by C. T. Russell, E. R. Priest, and L. C. Lee, pp. 1-22, American Geophysical Union, 1990.

Rème, H., et al., First multi-spacecraft ion measurements in and near the Earth's magnetosphere with the identical Cluster Ion Spectrometry (CIS) experiment, Ann. Geophys., 19, 1303, 2001.

Schindler, K., A theory of the substorm mechanism, J. Geophys. Res., 79, 2803, 1974.

Sergeev, V. A., et al., A two-satellite study of nightside flux transfer events in the plasma sheet, Planet. Space Sci., 40, 1551, 1992.

Slavin, J. A., et al., Geotail observations of magnetic flux ropes in the plasma sheet, J. Geophys. Res., 108(A1), 1015, doi:10.1029/2002JA009557, 2003.

M. H. Acuna, D. H. Fairfield, J. Gjerloev, M. L. Goldstein, R. P. Lepping, and J. A. Slavin, Laboratory for Extraterrestrial Physics, NASA GSFC, Greenbelt, MD 20771, USA. ( james.a.slavin@gsfc.nasa.gov)

A. Balogh and M. Dunlop, Space and Atmospheric Physics, Imperial College, London SW7 2BZ, UK.

J. M. Bosqued and H. Reme, Centre d'Etude Spatiale des Rayonnements, B.P. 4346, Toulouse, France.

A. Fazakerley and C. J. Owen, Mullard Space Science Laboratory, Holmbury St. Mary, Dorking RH5 6NT, UK.

K. Khurana and M. G. Kivelson, Institute of Geophysics and Planetary Physics, University of California, Los Angeles, CA 90024, USA. 